\title{
Percutaneous Endoscopic Interlaminar Discectomy for L5-S1 Axillary-Type Disc Herniation, with Surgeon Stand on an Opposite Side of Disc Protrusion to Perform
}

\section{Zhiqiang Ren}

Shenzhen Traditional Chinese Medicine Hospital

Shenghua He ( $\nabla$ rzq13026@126.com)

shenzhen hospital of chinese medicine

Jiao Li

shenzhen hospital of guangzhou university of chinese medicine

Jianshen Zhao

Shenzhen Traditional Chinese Medicine Hospital

\section{Xiufang Zhang}

Shenzhen Traditional Chinese Medicine Hospital

Juyi Lai

Shenzhen Traditional Chinese Medicine Hospital

Zhiming Lan

Shenzhen Traditional Chinese Medicine Hospital

Technical advance

Keywords: percutaneous endoscopic interlaminar discectomy, interlaminar approach, lumbar disc herniation

Posted Date: May 7th, 2021

DOI: https://doi.org/10.21203/rs.3.rs-496702/v1

License: (c) (i) This work is licensed under a Creative Commons Attribution 4.0 International License. Read Full License 


\section{Abstract}

Background: It is widely acceptable that surgeon stand on the same side of the disc protrusion to perform PEID. Few study focus on PEID being performed when surgeon stand on the opposite side of the disc protrusion. The aim is to investigate the feasibility and efficacy of an different approach that surgeon stood on the opposite side of the disc protrusion to perform PEID.

Methods: A total of 40 patients with radiculopathy due to L5-S1 disc herniation underwent PEID were included in this study. All surgeries were performed by a senior surgeon who stood on the opposite side of the disc protrusion. The pre- and postoperative VAS, JOA, ODI and modified MacNab scores, as well as postoperative complications were collected.

Results: All patients were followed up and the average follow-up period was 12.7 months. The VAS, JOA and ODI score were significantly improved after surgery. The excellent and good rate was $90 \%$ of the patients according to MacNab criteria. Recurrence without reoperation was observed at 6 months in 2 patient. No patient had major complications at the last follow-up except three cases who complained of transient lower limbs numbness.

Conclusion: In this study, we found axillary herniated discs could be removed easily when surgeon stood on the opposite side of the disc protrusion. A working cannula was introduced into the epidural space at an less steeper angle to the skin, which can effectively reduce the risk of dura sac injury.

\section{Background}

Percutaneous endoscopic interlaminar discectomy(PEID) is a common surgical treatment for L5S1lumbar disc herniation $(\mathrm{LDH})^{1-2}$. Although many studies have shown the efficacy of PEID with excellent clinical outcomes, nerve injury during surgery remains an important problem, espically the injury of cauda equina injury. It is widely acceptable that surgeon stand on the same side of the disc protrusion to perform PEID. In such a situation, cannula were inserted into spinal canal with an steep angle close to vertical. Since right below working cannula was the dura sac, surgeon need to be very careful about endoscopic insertion. There is a concern in PEID with the possibility of cauda equina injury or durotomy because of excessive neural retraction and manipulation of neural tissue, especially the working channel was unexpectedly inserted into the spinal canal for an inexperienced surgeon.Few study focus on PEID being performed when surgeon stand on the opposite side of the disc protrusion. In order to reduce the risk of nerve injure caused by working cannula, we made an effort to stand on the opposite side of disc protrusion to perform PEID, making the working cannula be inserted not vertically but at a less steeper angle into spinal canal, which can effectively avoid the unexpected and sudden injury of dura mater. In this study, we aimed to describe a different surgical approach to perform PEID to reduce the risk of cauda equina injury. We assessed the feasibility and efficacy of the surgical approach.

\section{Methods}


A database of cases with L5-S1 LDH undergoing PEID surgery was reviewed from January 2017 to March 2018. There were 40 patients included in the study. All patients underwent the same surgical procedure on levels L5-S1. The patients provided informed consent for the publication of their clinical and radiological data. This study was approved by medical ethical committee of our hospital.

\section{Inclusion and exclusion criteria}

Inclusion criteria included: Single-level symptomatic LDH (limited to L5-S1) with typical symptoms of low back pain and leg pain; The herniated disc was classified as axillary type; Physical examination showed positive Lasegue's sign, motor weakness, or sensory changes; Conservative treatment was ineffective for more than 4 weeks.

Exclusion criteria: Patients with multilevel disc herniation, severe spinal stenosis, cauda equina syndrome, spondylolisthesis or any other significant lumbar spinal instability were not considered eligible for the study. Those who had central disc herniation were also excluded.

\section{Surgical procedure}

All surgeries were performed under general anesthesia by a senior surgeon. Intraoperative neurophysiological monitoring were monitored throughout surgery to assess the function of the spinal cord and provides early detection to prevent latrogenic neurological deficits. Patients were placed on a radiolucent table in the prone position. The diseased segment was located under fluoroscopic guidance, and the needle insertion point was marked. The surgeon stood at the opposite side of disc protrusion and a skin incision of $6 \mathrm{~mm}$ was made in the mid-portion of the lateral half of the lesion side of the interlaminar window. The skin incision was approximately $1.0 \mathrm{~cm}$ lateral from the midline at the target disc level. Then a dilator was inserted to reach the intervertebral space under fluoroscopic guidance. The working cannula was introduced over the dilator and the irrigation system was connected. Ligmentum flavum was split with blunt dissector and scissors and enlarged with working tube under endoscopic visualization. After identification of epidural fat and dura sac, the spinal endoscope was advanced into the spinal canal. The working cannula and the horizontal plane form a angle of 15-30 degrees to avoid dura sac compression. Discectomy was performed at the axilla of the traversing nerve root. We then rotated the working cannula to further clean residual disc tissues at the shoulder region. In addition, the bipolar radiofrequency electrode was used to regulate heamostasis and mange the rupture in the annulus fibrosus. After confirmation of sufficient nerve root decompression and hemostasis, the endoscope and working tube were removed. A skin was closed with 3-0 nylon. The $5 \mathrm{mg}$ dexamethasone and analgesic drugs were given as symptomatic treatment after operation. Patients were discharged the next day and encouraged to do back muscles exercises. They were scheduled to visit the clinic at 1, 3 and 12 months after operation.

\section{Clinical and radiological outcomes assessment}


Clinical outcomes, such as VAS, JOA, ODI scores pre-operatively, 1 month and one year after surgery were collected. The improvement in symptoms was evaluated by calculating the difference between the VAS, JOA and ODI at the final follow-up and at presentation. Macnab standard ${ }^{3}$ [poor, fair, good or excellent] was used to evaluate patients' satisfaction with the surgery. After the operation, we obtained an immediate postoperative MRI for all patients and confirmed evacuation of LDH on MRI. The conditions of complication during perioperative period were recorded. Recurrence was classified as disc herniation on the same side and level as the primary operative site after successful initial removal of the protruding disc and a pain-free interval after surgery.

\section{Statistics}

SPSS 19.0 statistical software was used for statistical analysis. The measurement data were expressed by $(x \pm s)$, and the paired $T$ test was used for the comparison before and after operation. The difference was statistically significant with $\mathrm{P}<0.05$.

\section{Results}

\section{General characteristics of the patients}

A total of 40 patients were included in the study, including 17 males and 23 females, with an average age of 44.6 years (range, 19-76). The mean duration of history was 8.9 months, the mean operative time was 56.5 minutes and the mean blood loss was negligible. All patients were identified as axillary-type disc herniations. All patients were followed up at least 1 year and followed-up with an average period of 12.7 months(range, 12 to 18 months).The demographic data of patients are shown in Table 1.

\section{Surgical outcomes}

All patients underwent PEID smoothly, and showed relief of their symptoms after the operation. Clinical improvement was excellent. Preoperative average of VAS for low back was $6.05 \pm 1.36,3.50 \pm 1.41$ immediately after surgery, $2.30 \pm 1.14$ at 3 months and $1.83 \pm 1.22$ at the final follow-up examination. The VAS for lower limbs also improved after surgery $(6.78 \pm 1.46$ pre-operatively, $3.20 \pm 1.34$ immediately postoperatively, $2.03 \pm 0.95$ at 3 months and $1.23 \pm 1.53$ at the final follow-up). The mean value for the ODI improved from $69.28 \pm 13.87$ at presentation to $20.40 \pm 9.86$ at the final follow-up, JOA scale improved from $11.65 \pm 2.73$ to $22.08 \pm 2.95$. All scales exhibited statistically significant improvement $(p<0.01)$. Based on the modified MacNab criteria, the final outcome was found to be excellent in 30 cases, good in 6 cases, fair in 2 case and poor in 2 cases. The excellent and good rate was $90 \%$. Recurrence without reoperation was observed at final follow up in 2 patient. Surgical outcomes are shown in Table 2.

\section{Radiological Outcomes}

MRI examinations was performed at the second day after operation. The preoperative and postoperative radiologic findings revealed that the decompression of the herniated nucleus pulposus was complete 
except one patient showed symptomatic residual disc material,whose leg pain persisted postoperatively but disappeared gradually after 3 months without a second operation.

\section{Complications}

We observed no major complications in this case series after surgery. No patient had cauda equina syndrome, nerve root injury, cerebrospinal fluid leakage, infections, or major blood vessel at the last follow-up.Three patients who complained of transient lower limbs numbness after surgery were observed in our study. Their symptomes disappeared gradually after 3 months.

\section{Discussion}

$\mathrm{LDH}$ is one of the most common diseases seen in department of orthopaedics ${ }^{4}$. Most herniation sites are located at $\mathrm{L} 4 / 5$ and $\mathrm{L} 5 / \mathrm{S} 1^{5}$. Percutaneous endoscopic transforaminal discectomy (PETD) has been widely used in L4-5 LDH over the past several years. Unlike L4-5, L5-S1 has its own anatomical characteristics ${ }^{6-7}$, such as a high iliac crest and a large $L 5$ transverse process, which limit the transforaminal access to the L5-S1 disc space. PEID is a minimally invasive technique that can directly reach the spinal canal via the interlaminar space, which avoids factors such as a high iliac crest and transverse process variation and has obvious advantages in the treatment of L5-S1 LDH. PEID is one of the most sophisticated operative procedures for the treatment of L5-S1 LDH. Ruetten ${ }^{8}$ et al. were the first to perform intervertebral disc discectomy and decompression by creating an intervertebral foramen in the vertebral canal between the upper and lower vertebral discs. Compared with open discectomy, PEID offers the advantages of small incision size, short hospital stay, less blood loss, less destruction of surrounding tissues, and faster functional recovery ${ }^{9}$.

For PETD, transforaminal access involves passing endoscopes and instruments through a cannula placed safely in the Kambin's safe zone to avoid nerve injury ${ }^{10}$. However, PEID beginners should first realize there is no such "safe zone". The procedure from the initial punctures to the final placement of working cannel is the most difficult and critical part of the surgery ${ }^{11}$. Also, it is the most likely part to injure nerve. Sometimes, the working channel was unexpectedly inserted into the spinal canal through the widened interlaminar space, which could caused dural tear or nerve root injury. There is a concern in PEID with the possibility of cauda equina injury or durotomy because of excessive neural retraction and manipulation of neural tissue, especially for an inexperienced surgeon.

In order to reduce the risk of nerve injure, many spine surgeons had tried a lot of ways and it worked really well. Ruetten ${ }^{12}$ suggsted that ligamentum flavum was firstly opened with endoscopic scissors and forceps, then the opening was enlarged with working tube. After identification of epidural fat and neural tissue, the spinal endoscope was safely advanced into the spinal canal. Choi ${ }^{13}$ recommended that a dilator was inserted under fluoroscopy to reach the intervertebral space and the use of dilators from small to large in diameter to prevent nerve injury. Nicoletti ${ }^{14}$ advocated the incision of ligamentum flavum and placement of working catheter should under the supervision of complete endoscopy, which can ensure 
safety at any step of endoscopic procedures. Coi et al ${ }^{15}$ suggested that the interlaminar approach was safe for reaching the axillary space. For a axillary herniation, the nerve root was easily identified using a nerve dissector along the lateral recess under endoscopy. Because the nerve root travels horizontally at the L5/S1 level, it is safe to manage the axillary lesion. The oblique distal opening of the sheath should be toward the midline of the spinal canal to effectively expose the lesion and protect the nerve root and dura mater. Sufficient attention should be paid to intraoperative protection of the dura mater. In order to prevent cerebrospinal fluid leakage, care should be taken not to tear the dura during rotation. In this study, we tried to stand on the opposite side of disc protrusion to perform PEID. We found axillary herniated discs could be removed easily when surgeon stood on the opposite side of the disc protrusion. A working cannula was introduced into the epidural space at an oblique angle to the horizontal plane, which can directly access the axillary herniated disc and remove the disc fragment with minimal manipulation of the neural structure. The skin entry point of paraspinal approach is medial and the angle to the skin is less steeper compared with angle of the conventional approach, which can effectively reduce the risk of dura sac injury.

Excellent outcomes were observed after surgery. We found an improvement of VAS, ODI and JOA, which were significantly different from those in preoperation, suggesting that PEID would be beneficial for L5-S1 disc herniations to relieve pain. The modified MacNab criteria were calculated at the last follow-up.The excellent and good portion in MacNab accounted for $90 \%$, which could also explain the good results. In our study, we report a recurrence rate of $5 \%$, which is similar with the previous reports ${ }^{16}$. No patient had cauda equina syndrome, nerve root injury, cerebrospinal fluid leakage, infections, or major blood vessel at the last follow-up. Leg pain persisted postoperatively in only one case. Three patients complained of transient lower-extremity dysesthesia after surgery, which disappeared gradually after 3 month without any intervention. Stretch injury of the nerve root during operation may be a main factor. Researchers ${ }^{17}$ compared the surgical outcomes of the interlaminar and the transforaminal approaches and found that postoperative lower-extremity dysesthesia occurred mainly during the interlaminar approach. Suess ${ }^{18}$ found that dysesthesia generally occurred during the early postoperative period, and resection of the lesion step-by-step under direct vision can reduce the risk of potential nerve root injury. We believe nerve root traction caused by the rotation of the working cannula may result in lower-extremity dysesthesia during the early postoperative period.

We are aware of several limitations to this study. First, the study was performed as an observational study, no control group has been included for comparison. Second, the number of patients was small, a larger number of cases was advisable.

\section{Conclusions}

Although, it is widely acceptable that surgeon stand on the same side of the disc protrusion to perform PEID, we found axillary herniated discs could be removed easily when surgeon stood on the opposite side of the disc protrusion. A working cannula was introduced into the epidural space at an less steeper angle to to the skin, which can directly access the axillary herniated disc and remove the disc fragment with 
minimal manipulation of the neural structure. According to this study,we believe the incidence of dura sac injury can be effectively reduced when surgeon stand on the opposite side of the disc protrusion to perform PEID.

\section{Abbreviations}

Although, it is widely acceptable that surgeon stand on the same side of the disc protrusion to perform PEID, we found axillary herniated discs could be removed easily when surgeon stood on the opposite side of the disc protrusion. A working cannula was introduced into the epidural space at an less steeper angle to to the skin, which can directly access the axillary herniated disc and remove the disc fragment with minimal manipulation of the neural structure. According to this study,we believe the incidence of dura sac injury can be effectively reduced when surgeon stand on the opposite side of the disc protrusion to perform PEID.

PEID-percutaneous endoscopic interlaminar discectomy;VAS-visual analogue scale; JOA-Japanese Orthopaedic Association; ODI-Oswestry Disability Index

LDH=Lumbar Disc Herniation; MRI=Magnetic Resonance Imaging

\section{Declarations}

Ethics approval and consent to participate: The study was approved by medical ethics committee of our hosptial.

Consent for publication: Manuscript is approved by all authors for publication.

Availability of data and material:The datasets used or analysed during the current study are available from the corresponding author on reasonable request.

Competing interests:The authors have no personal financial or institutional interests in any of the drugs, materials or devices described in this article.

\section{Funding:None}

Authors' contributions: Zhiqiang Ren contributed to the conception of the study;

Shenghua He performed the surgery; Jiao Li contributed significantly to analysis and manuscript preparation;Jianshen Zhao , Xiufang Zhang performed the data analyses and wrote the manuscript;Lai Juyi , Zhiming Lan helped perform the analysis with constructive discussions.

Acknowledgements: We thank all the operating room staff for their technical assistance and the medical records clerks who helped to collect patient data. 


\section{References}

1. Ruetten S, Komp M, Merk H, Godolias G. Use of newly developed instruments and endoscopes: Fullendoscopic resection of lumbar disc herniations via the interlaminar and lateral transforaminal approach. J Neurosurg Spine. 2007;6:521-30.

2. Ruetten S, Komp M, Godolias G. A New full-endoscopic technique for the interlaminar operation of lumbar disc herniations using 6-mm endoscopes:Prospective 2-year results of 331 patients. Minim Invasive Neurosurg. 2006;49:80-8.

3. MacNab I. Negative disc exploration. An analysis of the causes of nerve-root involvement in sixtyeight patients. J Bone Joint Surg Am. 1971;53:891-903.

4. Amin RM, Andrade NS, Neuman BJ. Lumbar disc herniation. Curr Rev Musculoskelet Med. 2017;10:507-16.

5. Reihani-Kermani H. Level-diagnosis of lumbar disc herniation. Iran J Med Sci,2003, 135-138.

6. Chen HT, Tsai CH, Chao SC, et al. Endoscopic discectomy of L5-S1 disc herniation via an interlaminar approach: Prospective controlled study under local and general anesthesia. Surg Neurol Int. 2011;2:93.23.

7. Chumnanvej S, Kesornsak W, Sarnvivad P, er al. Full endoscopic lumbar discectomy via interlaminar approach: 2-year results in Ramathibodi Hospital. J Med Assoc Thai 2011; 94:1465-1470.

8. Ruetten S, Komp M, Merk H, et al. Recurrent lumbar disc herniation after conventional discectomy: a prospective, randomized study comparing full-endoscopic interlaminar and transforaminal versus microsurgical revision. J Spinal Disord Tech. 2009;22:122-9.

9. Yeung AT, Tsou PM. Posterolateral endoscopic excision for lumbar disc herniation:Surgical technique, outcome, and complications in 307 consecutive cases. Spine (Phila Pa 1976). 2002;27:722-31.

10. Kambin P, Gellman H. Percutaneous lateral discectomy of the lumbar spine a preliminary report. Clin Orthop Relat Res. 1983;174:127-32.

11. Hoogland TT, Brekel-Dijkstra KVD, Schubert M, Miklitz B. Endoscopic transforaminal discectomy for recurrent lumbar disc herniation: a prospective, cohort evaluation of 262 consecutive cases. The Spine Journal. 2008;33(9):973-8.

12. Ruetten $\mathrm{S}, \mathrm{Komp} M$, Merk $\mathrm{H}$, et al. Surgical treatment for lumbar lateral recess stenosis with the fullendoscopic interlaminar approach versus conventional microsurgical technique: a prospective, randomized, controlled study. J Neurosurg Spine. 2009;10(5):476-85.

13. Choi G, Lee SH, Deshpande K, et al. Working channel endoscope in lumbar spine surgery. J Neurosurg Sci. 2014;58:77-85.

14. Nicoletti GF, Platania N, Albanese V. Smooth dissection of ligamentum flavum for lumbar microdiscectomy. Preliminary report of this personal technique. Surg Neurol. 2005;64:232-5.

15. Choi G, Lee SH, Raiturker PP, et al. Percutaneous endoscopic interlaminar discectomy for intracanalicular disc herniations at L5-S1 using a rigid working channel endoscope. Neurosergery. 
2006;58(1):59-68.

16. Ruetten S, Komp M, Merk H, Godolias G. Full-endoscopic interlaminar and transforaminal lumbar discectomy versus conventional microsurgical technique: A prospective, randomized, controlled study. Spine. 2008;33:931-9.

17. Choi KC, Kim JS, Ryu KS, et al. Percutaneous endoscopic lumbar discectomy for L5-S1 disc herniation: Transforaminal versus interlaminar approach. Pain Physician. 2013;16:547-56.

18. Suess $\mathrm{O}$, Brock $\mathrm{M}$, Kombos $T$. Motor nerve root monitoring during percutaneous transforaminal endoscopic sequestrectomy under general anesthesia for intra- and extraforaminal lumbar disc herniation. Zentralbl Neurochir. 2005;66:190-201.

\section{Tables}

Table 1. Clinical and epidemiological data $(\overline{\mathrm{x}} \pm \mathrm{s})$

\begin{tabular}{|ll|}
\hline Characteristic & Value \\
\hline Number of cases & 40 \\
\hline Gender(male:female) & $17: 23$ \\
\hline BMI $(\mathrm{kg} / \mathrm{m})$ & $22.35 \pm 2.47(17.7-30.4)$ \\
\hline Age(year) & $44.6 \pm 16.2(19-76)$ \\
\hline Type of disc herniation & axillary-type \\
\hline Duration of history(mo) & $8.9 \pm 7.2(1-24)$ \\
\hline Follow-up(mo) & $12.7 \pm 1.4(12-18)$ \\
\hline Operative time(min) & $56.5 \pm 12.7(32-89)$ \\
\hline Length of stay(d) & $3.3 \pm 0.8$ \\
\hline Recurrence & $2(5 \%)$ \\
\hline MacNab criteria & \\
\hline Excellent & $30(75 \%)$ \\
\hline Good & $6(15 \%)$ \\
\hline Fair & $2(5 \%)$ \\
\hline Poor & $2(5 \%)$ \\
\hline
\end{tabular}

Table 2 Surgical outcome $(\overline{\mathrm{x}} \pm \mathrm{s})$ 


\begin{tabular}{|c|c|c|c|c|}
\hline & \multirow[t]{2}{*}{ Preop } & Postop & Postop & Postop \\
\hline & & immediate & 3-month & 1 -year \\
\hline VAS for low back & $6.05 \pm 1.36$ & $3.50 \pm 1.41 \rrbracket$ & $2.30 \pm 1.14 \Delta$ & $1.83 \pm 1.22 \square$ \\
\hline VAS for lower limbs & $6.78 \pm 1.46$ & $3.20 \pm 1.34 \rrbracket$ & $2.03 \pm 0.95 \Delta$ & $1.23 \pm 1.53 \square$ \\
\hline JOA & $11.65 \pm 2.73$ & & & $22.08 \pm 2.95 \square$ \\
\hline ODI & $69.28 \pm 13.87$ & & & $20.40 \pm 9.86 \square$ \\
\hline $\begin{array}{l}\text { Preop=preoperat; } P \text { so } \\
\text { mean values }(P<0.01 \\
\text { values }(P<0.01) .\end{array}$ & $\begin{array}{l}\text { stoperative } \mathbb{\mathbb { }} \\
\text { mparison of } p \\
\text { son of preope }\end{array}$ & $\begin{array}{l}\text { arison of prec } \\
\text { erative and } 3 r \\
\text { e and last follc }\end{array}$ & $\begin{array}{l}\text { ative and imm } \\
\text { ths after surge } \\
\text { ip mean values }\end{array}$ & $\begin{array}{l}\text { ely after surgery } \\
\text { ean } \\
.01) \text {. }\end{array}$ \\
\hline
\end{tabular}

\section{Figures}

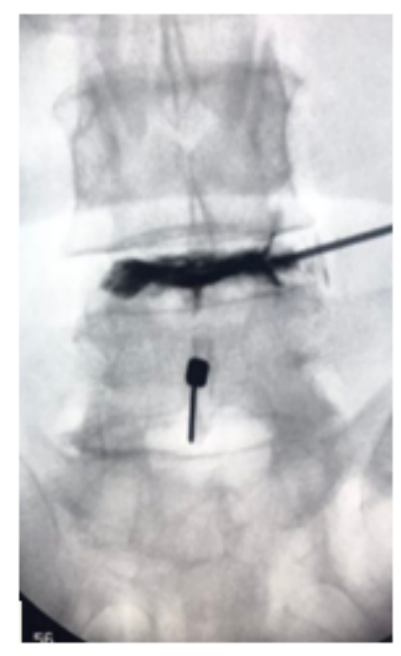

Figure 1

The puncture point was approximately $1.0 \mathrm{~cm}$ lateral from the midline at L5-S1 disc level. 


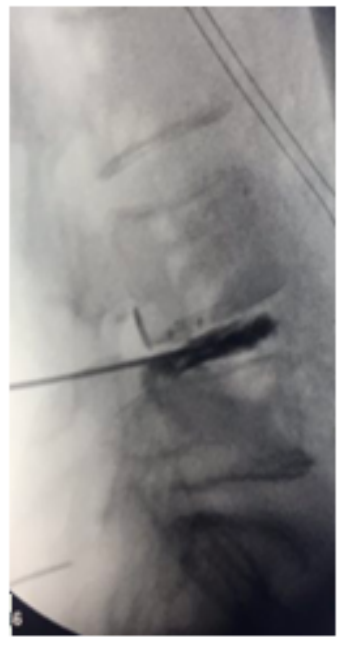

Figure 2

The puncture needle was inserted to reach the intervertebral space under fluoroscopic guidance

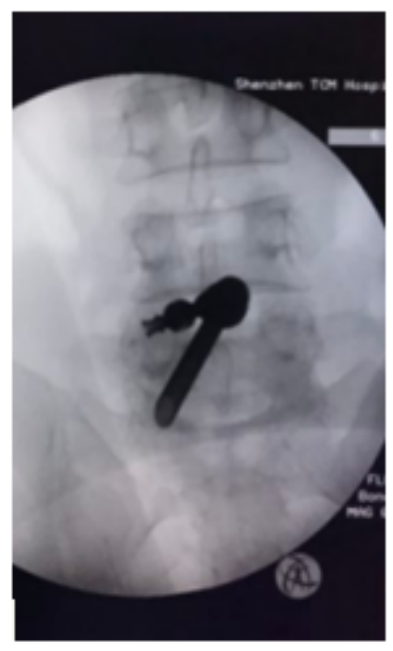

\section{Figure 3}

A working cannula was introduced into the epidural space at an less steeper angle to the skin compared with angle of the conventional approach. 


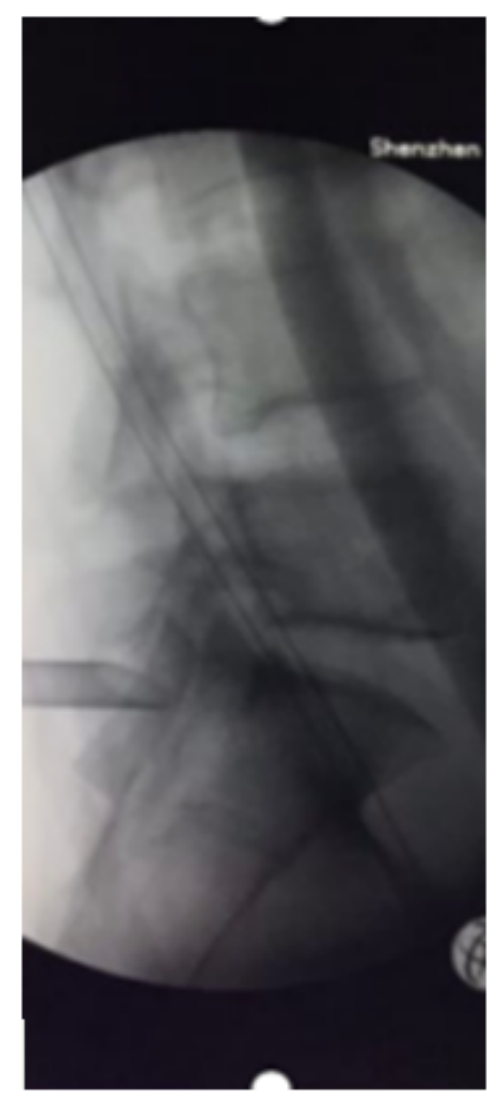

Figure 4

The working cannula was inserted to reach the intervertebral space under fluoroscopic guidance. 


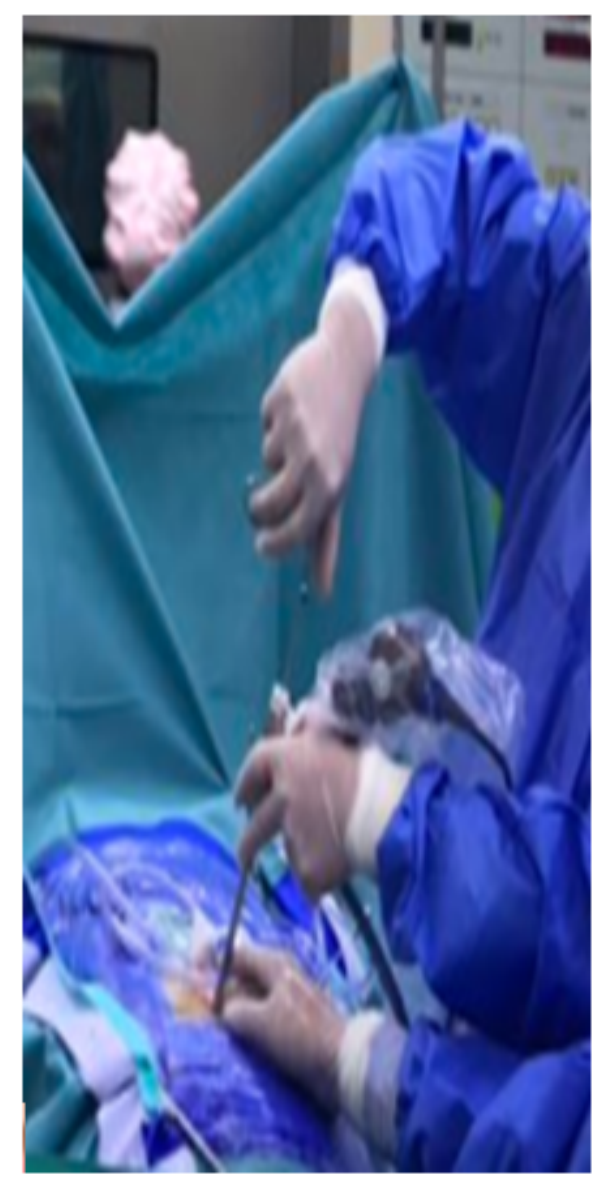

Figure 5

The surgeon stand at the opposite side of disc protrusion to perform PEID. A working cannula was introduced into the epidural space at an less steeper angle to to the skin, which can directly access the axillary herniated disc and remove the disc fragment with minimal manipulation of the neural structure. 


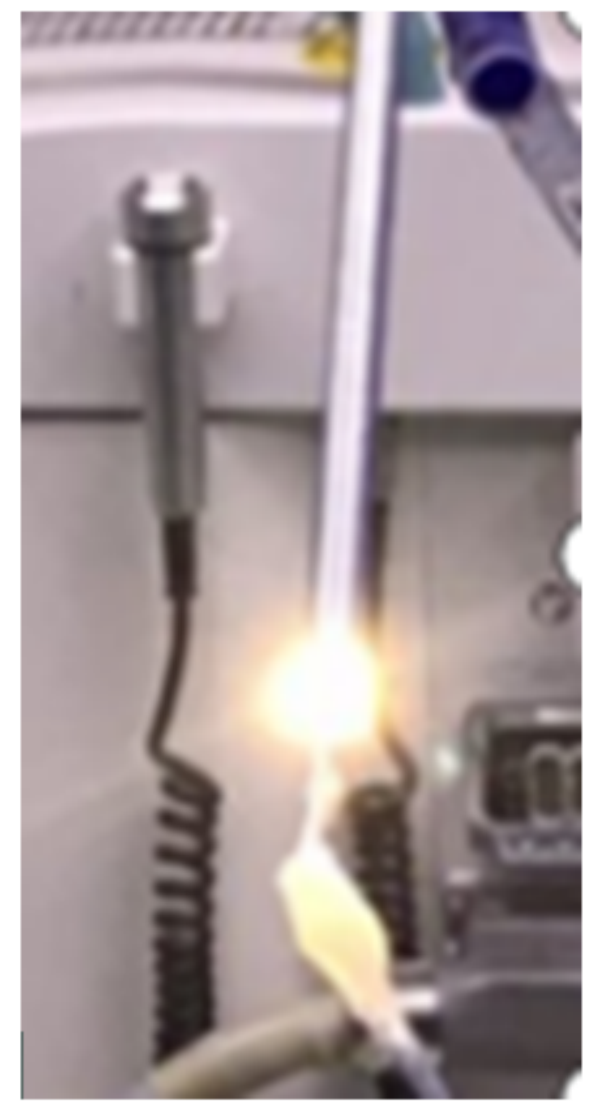

Figure 6

We found axillary herniated discs could be removed easily when surgeon stood on the opposite side of the disc protrusion.

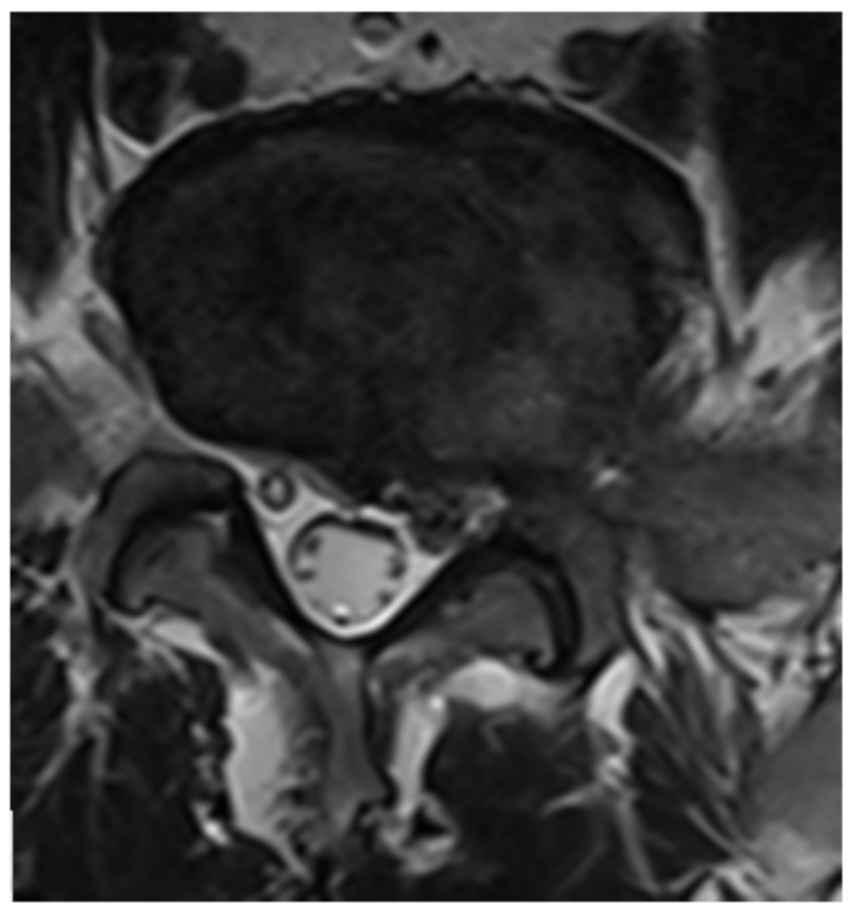




\section{Figure 7}

The herniated disc was classified as axillary type on MRI

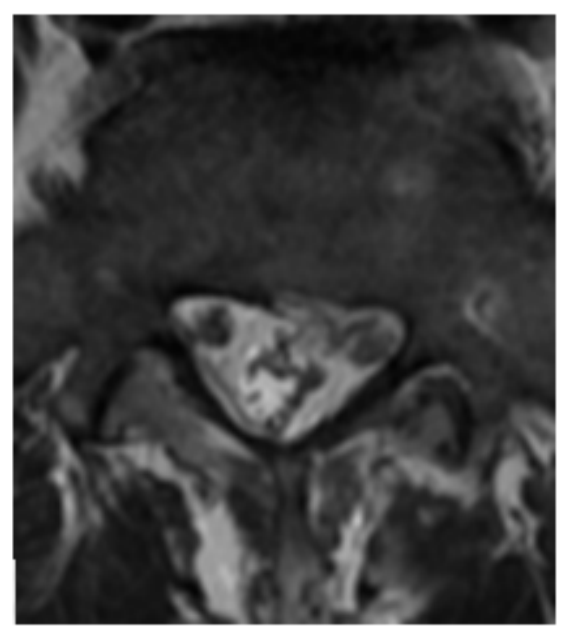

\section{Figure 8}

The preoperative and postoperative MRI findings revealed that the decompression of the herniated nucleus pulposus was complete. 